\title{
Erratum to: Convergence in Variation and Rate of Approximation for Nonlinear Integral Operators of Convolution Type
}

\author{
Laura Angeloni and Gianluca Vinti
}

Erratum to: Results. Math. (2006) 49:1-23

DOI 10.1007/s00025-006-0208-2

In the present erratum the authors want to mark out that the term $I_{2}$ of Theorem 1 (convergence theorem) in [1] has to be estimated in a different way. A similar problem occurs in Theorem 2 of [1], where in order to get the required result one has to assume, instead of (2) of the original paper (involving assumption $K_{w} .3$ ) of [1]), condition (I) below (involving assumption $\left.K_{w} .3\right)^{\prime}$ mentioned below). Since it is easy to see that the two assumptions $K_{w} .3$ ) and $\left.K_{w} .3\right)^{\prime}$ cannot be compared, in order to unify our approach, here we prove Theorem 1 by using $\left.K_{w} .3\right)^{\prime}$ even if, as mentioned in the Remark of Sect. 1, $\left.K_{w} .3\right)$ is still valid in order to prove the result. Similar reasonings hold also for the multidimensional case.

\section{Periodic Case}

For the notations we refer to [1]. Assumption $\left.K_{w} .3\right)$ of the original paper ([1]) has to be replaced by the following

$\left.\mathbf{K}_{\mathbf{w}} \cdot \mathbf{3}\right)^{\prime}$ denoted by $G_{w}(u):=H_{w}(u)-u, u \in \mathbb{R}, w>0$,

$$
\frac{V_{J}\left[G_{w}\right]}{m(J)} \rightarrow 0, \quad \text { as } w \rightarrow+\infty,
$$

The online version of the original article can be found under doi:10.1007/s00025-006-0208-2. 
uniformly with respect to every (proper) bounded interval $J \subset \mathbb{R}$, that is, for every $\varepsilon>0$ there exists $\bar{w}>0$ such that $\frac{V_{J}\left[G_{w}\right]}{m(J)}<\varepsilon$, for every $w \geq \bar{w}$ and for every bounded interval $J \subset \mathbb{R}$.

We remark that it is easy to provide examples of kernels which fulfill assumption $\left.K_{w} \cdot 3\right)^{\prime}$, besides all the other assumptions of our theory. For example, let us consider the kernel functions of Example 3 of [1], namely $K_{w}(t, u)=$ $L_{w}(t) H_{w}(u), t \in \mathbb{R}_{0}^{+}, u \in \mathbb{R}, w>0$, where $\left\{L_{w}(t)\right\}_{w>0}$ is an approximate identity,

$$
H_{w}(u)=\left\{\begin{array}{l}
u+\log \left(1+\frac{u}{w}\right), \quad 0 \leq u<1, \\
u+\log \left(1+\frac{1}{w u}\right), \quad u \geq 1,
\end{array}\right.
$$

and the definition of $H_{w}(u)$ is extended in odd-way for $u<0$. Then

$$
G_{w}(u)=\left\{\begin{array}{l}
\log \left(1+\frac{u}{w}\right), \quad 0 \leq u<1, \\
\log \left(1+\frac{1}{w u}\right), \quad u \geq 1,
\end{array}\right.
$$

and it is easy to see that $G_{w}(u)$ is increasing in $[0,1]$, decreasing in $[1,+\infty)$. Hence, for every interval $[a, b] \subset[0,1]$ there holds

$$
\frac{V_{[a, b]}\left[G_{w}\right]}{m([a, b])}=\frac{\log \left(1+\frac{b}{w}\right)-\log \left(1+\frac{a}{w}\right)}{b-a} \leq \frac{1}{w} \rightarrow 0
$$

as $w \rightarrow+\infty$, and, for every $[a, b] \subset[1,+\infty)$,

$$
\frac{V_{[a, b]}\left[G_{w}\right]}{m([a, b])}=\frac{\log \left(1+\frac{1}{a w}\right)-\log \left(1+\frac{1}{b w}\right)}{b-a} \leq \frac{1}{w a b} \leq \frac{1}{w} \rightarrow 0
$$

as $w \rightarrow+\infty$. If $[a, b] \subset \mathbb{R}$ is such that $0 \leq a<1<b$, it is sufficient to notice that $V_{[a, b]}\left[G_{w}\right]=V_{[a, 1]}\left[G_{w}\right]+V_{[1, b]}\left[G_{w}\right]$. This implies that $\left.K_{w} .3\right)^{\prime}$ holds.

It is possible to prove the following

Lemma 1. Let $f \in B V_{2 \pi} \cap C_{2 \pi}^{0}$, where $C_{2 \pi}^{0}$ denotes the space of continuous functions over $[-\pi, \pi]$. If $\left.K_{w} .3\right)^{\prime}$ is satisfied, then

$$
V_{2 \pi}\left[H_{w} \circ f-f\right] \rightarrow 0, \quad \text { as } \quad w \rightarrow+\infty .
$$

Proof. Since $f \in C_{2 \pi}^{0} f$ has at most countably infinitely many proper points of maximum/minimum ([2]). Let us consider the most general case in which $f$ has countably infinitely many proper points of maximum/minimum, that we will denote as $\bar{x}_{i}, i=0,1,2, \ldots$. Let $D=\left\{x_{0}=-\pi, x_{1}, \ldots, x_{n}=\pi\right\}$ be a division of the interval $[-\pi, \pi]$ and let $\widetilde{D} \equiv\left\{y_{0}, y_{1}, \ldots\right\}$ be the (infinite) division obtained adding the points $\bar{x}_{i}$ to $D$. Without any loss of generality let us assume that $\lim _{i \rightarrow+\infty} \bar{x}_{i}=\pi$ (in the other cases it is sufficient to split the interval) and that $\bar{x}_{0} \equiv x_{0}=-\pi$ (if $\bar{x}_{0}>-\pi$, then $f$ is constant in $\left[-\pi, \bar{x}_{0}\right]$ and so $\left.V_{2 \pi}[f]=V_{\left[\bar{x}_{0}, \pi\right]}[f]\right)$. Then obviously $V_{2 \pi}[f]=\sum_{i=1}^{+\infty}\left|f\left(\bar{x}_{i}\right)-f\left(\bar{x}_{i-1}\right)\right|$. 
Hence in each of the intervals $A_{i}:=\left[\bar{x}_{i-1}, \bar{x}_{i}\right], i=1,2, \ldots, f$ is monotone, and so

$$
V_{A_{i}}\left[H_{w} \circ f-f\right] \leq V_{I_{i}}\left[G_{w}\right], \quad i=1,2, \ldots,
$$

where $I_{i}:=\left[\min \left\{f\left(\bar{x}_{i-1}\right), f\left(\bar{x}_{i}\right)\right\}, \max \left\{f\left(\bar{x}_{i-1}\right), f\left(\bar{x}_{i}\right)\right\}\right]$. By $\left.K_{w} \cdot 3\right)^{\prime}$, for a fixed $\varepsilon>0$, there exists $\bar{w}>0$ such that $V_{I_{i}}\left[G_{w}\right] \leq \varepsilon m\left(I_{i}\right)$, for every $w \geq \bar{w}$ and $i=1,2, \ldots$, and so

$$
\begin{aligned}
\sum_{i=1}^{+\infty}\left|\left(H_{w} \circ f-f\right)\left(y_{i}\right)-\left(H_{w} \circ f-f\right)\left(y_{i-1}\right)\right| & \leq \sum_{i=1}^{+\infty} V_{A_{i}}\left[H_{w} \circ f-f\right] \\
& \leq \sum_{i=1}^{+\infty} V_{I_{i}}\left[G_{w}\right] \\
& \leq \varepsilon \sum_{i=1}^{+\infty} m\left(I_{i}\right)=\varepsilon V_{2 \pi}[f] .
\end{aligned}
$$

Then, passing to the supremum over all the possible divisions of $[-\pi, \pi]$, we obtain that, for every $\varepsilon>0$, there exists $\bar{w}>0$ such that for every $w \geq \bar{w}$, $V_{2 \pi}\left[H_{w} \circ f-f\right] \leq \varepsilon V_{2 \pi}[f]$, and so the thesis follows, since by assumption $f \in B V_{2 \pi}$.

Remark. We remark that, in order to obtain the previous convergence result, it is sufficient to assume condition $K_{w} .3$ ) of the original paper (obviously using a different proof). However, since a condition of the form $\left.K_{w} .3\right)^{\prime}$ is needed in order to obtain the order of approximation and all the further results in the multidimensional frame, for a sake of simplicity we use directly condition $\left.K_{w} \cdot 3\right)^{\prime}$.

Using Lemma 1, the proof of Theorem 1 of the original paper follows. Indeed, it is sufficient to replace the estimate of $I_{2}$ (page 10, line 9) with the following

$$
I_{2} \leq \int_{-\pi}^{\pi}\left|L_{w}(t)\right| V_{2 \pi}\left[H_{w} \circ f-f\right] d t \leq A V_{2 \pi}\left[H_{w} \circ f-f\right]
$$

by assumption $K_{w} .1$ ), and so, by Lemma $1, I_{2} \leq A \varepsilon$, for sufficiently large $w>0$.

About the order of approximation, in a similar fashion, in Theorem 2 of the original paper instead of condition (2) we have to assume that

$$
\frac{V_{J}\left[G_{w}\right]}{m(J)}=O\left(\xi\left(w^{-1}\right)\right), \quad w \rightarrow+\infty,
$$

uniformly with respect to every (proper) bounded interval $J \subset \mathbb{R}$, i.e., there exists an absolute constant $M>0$ and $\bar{w}>0$ such that for every $w \geq \bar{w}$ and for every bounded interval $J \subset \mathbb{R}, \frac{V_{J}\left[G_{w}\right]}{m(J)} \leq M \xi\left(w^{-1}\right)$. It is not difficult to 
provide examples of kernels which satisfy (I): among them, for example, the family of kernel functions of Example 3 of the original paper (i.e., the above example of the present section) satisfy the above condition with $\xi(w)=w^{\alpha}$, $0<\alpha \leq 1$. Moreover, in the estimate of $V_{2 \pi}\left[T_{w} f-f\right]$ (page 12, line 8), instead of the term $A V_{J}\left[G_{w}\right]$ we now have $A V_{2 \pi}\left[H_{w} \circ f-f\right]$. As concerns this term, it is not difficult to see that, following the same reasoning of Lemma 1, by assumption (I), $V_{2 \pi}\left[H_{w} \circ f-f\right]=O\left(\xi\left(w^{-1}\right)\right)$, as $w \rightarrow+\infty$.

\section{Multidimensional Case}

We refer again to the original paper for the notations that we shall use in the multidimensional case. In an analogous way to the periodic case, assumption $\left.K_{w} .3\right)$ has to be replaced by $\left.K_{w} .3\right)^{\prime}$ and it is now possible, with this condition, to prove the following convergence result:

Lemma 2. Let $f \in A C\left(\mathbb{R}^{N}\right)$. If $\left.K_{w} .3\right)^{\prime}$ is satisfied, then

$$
V\left[H_{w} \circ f-f\right] \rightarrow 0, \text { as } w \rightarrow+\infty .
$$

Proof. Let $I=\prod_{i=1}^{N}\left[a_{i}, b_{i}\right]$ and let $\left\{J_{1}, \ldots, J_{m}\right\}$ be a partition of $I$, with $J_{k}=\prod_{j=1}^{N}\left[{ }^{(k)} a_{j},{ }^{(k)} b_{j}\right], k=1,2, \ldots, m$. Since $f \in A C\left(\mathbb{R}^{N}\right)$, for every $k=$ $1, \ldots, m$ in particular $f\left(x_{j}^{\prime}, \cdot\right)$ is continuous in $\left[{ }^{(k)} a_{j},{ }^{(k)} b_{j}\right]$ for almost every $x_{j}^{\prime} \in\left[{ }^{(k)} a_{j}^{\prime},{ }^{(k)} b_{j}^{\prime}\right]$ and for every $j=1, \ldots, N, w>0$. Hence, in a similar way to Lemma 1 , using $\left.K_{w} .3\right)^{\prime}$ it is possible to prove that, for every fixed $\varepsilon>0$ there exists $\bar{w}>0$ (depending only on $\varepsilon$ ) such that

$$
V_{\left[{ }^{(k)} a_{j},{ }^{(k)} b_{j}\right]}\left[\left(H_{w} \circ f-f\right)\left(x_{j}^{\prime}, \cdot\right)\right] \leq \varepsilon V_{\left[{ }^{(k)} a_{j},{ }^{(k)} b_{j}\right]}\left[f\left(x_{j}^{\prime}, \cdot\right)\right]
$$

for every $w \geq \bar{w}$. Then, for $w \geq \bar{w}$ there holds

$$
\begin{aligned}
\Phi_{j}\left(H_{w} \circ f-f, J_{k}\right) & =\int_{(k) a_{j}^{\prime}}^{(k) b_{j}^{\prime}} V_{\left[(k) a_{j},{ }^{(k)} b_{j}\right]}\left[\left(H_{w} \circ f-f\right)\left(x_{j}^{\prime}, \cdot\right)\right] d x_{j}^{\prime} \\
& \leq \varepsilon \int_{(k)}^{(k) b_{j}^{\prime}} V_{\left[{ }_{j}^{\prime}(k) a_{j},{ }^{(k)} b_{j}\right]}\left[f\left(x_{j}^{\prime}, \cdot\right)\right] d x_{j}^{\prime}=\varepsilon \Phi_{j}\left(f, J_{k}\right),
\end{aligned}
$$

for every $j=1, \ldots, N$. Hence $\Phi\left(H_{w} \circ f-f, J_{k}\right) \leq \varepsilon \Phi\left(f, J_{k}\right)$ and, passing to the supremum over all the possible partitions of $I, V_{I}\left[H_{w} \circ f-f\right] \leq \varepsilon V_{I}[f]$, for all $I \subset \mathbb{R}^{N}$. This implies that

$$
V\left[H_{w} \circ f-f\right] \leq \varepsilon V[f]
$$

for every $w \geq \bar{w}$, which completes the proof, since by assumption in particular $f \in B V\left(\mathbb{R}^{N}\right)$. 
Now, Theorem 3 of the original paper follows, replacing the estimate of $V\left[T_{w} f-f\right]$ with the following

$$
\begin{aligned}
V\left[T_{w} f-f\right] \leq & \int_{0 \leq|\mathrm{t}| \leq \delta}\left|L_{w}(\mathrm{t})\right| V\left[\left(H_{w} \circ f\right)(\cdot-\mathrm{t})-\left(H_{w} \circ f\right)(\cdot)\right] d \mathrm{t} \\
& +2 K V[f] \int_{|\mathrm{t}| \geq \delta}\left|L_{w}(\mathrm{t})\right| d \mathrm{t} \\
& +V\left[H_{w} \circ f-f\right] \int_{\mathbb{R}^{N}}\left|L_{w}(\mathrm{t})\right| d \mathrm{t}+\left|\bar{A}_{w}-1\right| V[f] \\
= & : J_{1}+J_{2}+J_{3}+J_{4},
\end{aligned}
$$

and taking into account that, by Lemma $2, J_{3} \leq A \varepsilon$. We finally remark that, following this proof, it is no more necessary to assume that $f \in L^{\infty}\left(\mathbb{R}^{N}\right)$ and so Theorem 3 of the original paper holds assuming only that $f \in A C\left(\mathbb{R}^{N}\right)$.

About the order of approximation, as before assumption (2) of Theorem 2 (used in Theorem 4) has to be replaced with condition (I) and estimate (7) has to be modified in a similar fashion to the periodic case, taking into account that, as in the proof of Lemma 2, it can be proved that $V\left[H_{w} \circ f-f\right]=O\left(\xi\left(w^{-1}\right)\right)$, as $w \rightarrow+\infty$. We remark that also in Theorem 4 of the original paper the assumption that $f \in L^{\infty}\left(\mathbb{R}^{N}\right)$ can be dropped.

\section{References}

[1] Angeloni, L., Vinti, G.: Convergence in variation and rate of approximation for nonlinear integral operators of convolution type. Results Math. 49(1-2), 1-23 (2006)

[2] Hobson, E.W.: The theory of functions of a real variable and the theory of Fourier's series. Dover Publications, New York (1957)

Laura Angeloni and Gianluca Vinti

Dipartimento di Matematica e Informatica

Università degli Studi di Perugia

Via Vanvitelli, 1

06123 Perugia

Italy

e-mail: angeloni@dmi.unipg.it;

mategian@unipg.it 